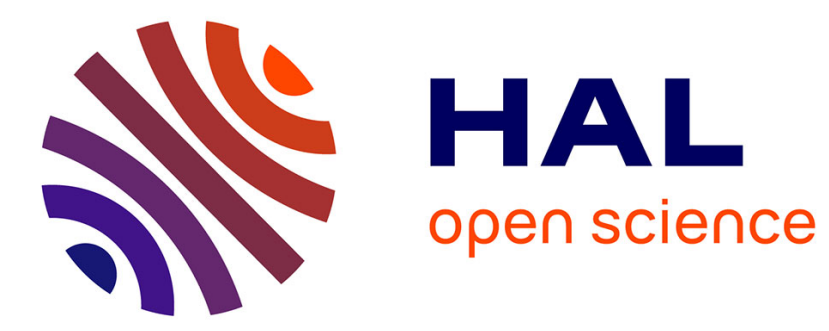

\title{
On the use of NURBS functions for displacement derivatives measurment by digital image correlation
}

Julien Réthoré, Thomas Elguedj, Pierre Simon, Michel Coret

\section{To cite this version:}

Julien Réthoré, Thomas Elguedj, Pierre Simon, Michel Coret. On the use of NURBS functions for displacement derivatives measurment by digital image correlation. Experimental Mechanics, 2010, 50

(7), pp.1099-1116. 10.1007/s11340-009-9304-z . hal-00517490

\author{
HAL Id: hal-00517490 \\ https://hal.science/hal-00517490
}

Submitted on 14 Sep 2010

HAL is a multi-disciplinary open access archive for the deposit and dissemination of scientific research documents, whether they are published or not. The documents may come from teaching and research institutions in France or abroad, or from public or private research centers.
L'archive ouverte pluridisciplinaire HAL, est destinée au dépôt et à la diffusion de documents scientifiques de niveau recherche, publiés ou non, émanant des établissements d'enseignement et de recherche français ou étrangers, des laboratoires publics ou privés. 


\title{
On the use of NURBS functions for displacement derivatives measurement by digital image correlation
}

\author{
Julien Réthoré · Thomas Elguedj . \\ Pierre Simon • Michel Coret
}

Received: date / Accepted: date

\begin{abstract}
In this paper, we propose to investigate the potential improvement of using Non-Uniform Rational B-Spline (NURBS) functions for displacement measurements by digital image correlation (DIC). The aim is at improving the performance of DIC to capture with low uncertainty and low noise levels not only the displacement field but also its derivatives. Indeed, when the displacement field is used to feed constitutive law identification procedures, displacement derivatives are required and thus may be measured with robustness. Two examples illustrate the potential of NURBS for DIC: a compressive test on a wood sample and a bending test on a steel beam. For the latter, beam kinematics are adopted and NURBS are used in order to capture the variation of the curvature (second derivative of the displacement) along the beam axis. For these two examples, an error study based on a decomposition of the error into
\end{abstract}

LaMCoS, INSA Lyon / CNRS / Universit de Lyon

20 Avenue Albert Einstein, 69621 VILLEURBANNE, FRANCE Tel.: +33472438787

Fax: +33472438206

E-mail: julien.rethore@insa-lyon.fr 
the correlation error and the interpolation error, is carried out and shows the great potential of NURBS functions for DIC.

Keywords Digital Image Correlation · Beam · B-splines

\section{Introduction}

Digital Image Correlation (DIC) is now one of the most popular full-field measurement techniques not only in the accademic world but also in industry. Since the pioneering paper by Sutton et al (1983), DIC has been successfully used in a wide range of applications. If we focus on constitutive model identification, the natural quantity that is obtained by (DIC), i.e. displacement, has to be post-processed to extract strains. This step is anything but obvious and has a strong influence on the identification procedure. The usual approach to DIC is based on a local independent pattern matching. Thus the strain field is reconstructed from the measured displacement at a set of measurement points (the pattern centers). A common technique consists in fitting the displacement field on a finite element (FE) discretization based on a independent mesh so that strains can be computed using FE shape functions. Note that further filtering is commonly used at this step in order to reduced noise levels. Even though an established practice, this may deteriorate the information contained in the measurement.

Recently, Besnard et al (2006) proposed a global approach to solve the optical flow equation. The main advantage of this formulation is that arbitrary basis functions can be used to describe the searched displacement. Initially, FE were proposed in Besnard et al but Williams' series (Roux and Hild 2006) or eXtended FE (Réthoré et al 2007a) have also been used in this framework. When FE are chosen, the approach appears as extremely robust as it ensures the continuity of the displacement over the 
entire domain of interest and thus decreases the under-determination of the optical flow equation. Further, as the same functions are used for measurement and identification, no fitting procedure is needed and the strains are directly derived from the measured displacement.

The tuning parameter of FE DIC is the element size: not too small to limit the impact of noise but not too big to capture the variation of the displacement. There is thus a compromise between correlation error that arises from the ill-posed nature of the problem, and interpolation error when the actual displacement cannot be interpolated with the chosen basis functions. One of the main advantages of full field measurement is that non conventional tests with non-homogeneous strain states can be analysed. Further, for a robust identification of damage laws or elastic-plastic constitutive laws, non-homogeneous, up to localization, strain states are required so that the wider range of strain states is explored. One thus needs to capture localized phenomena and a small element size is desired. A small element size means high noise levels and thus a less robust identification. To solve this conflict, one would like to reduce interpolation error without increasing the correlation error i.e. without increasing the ill-posedness of the problem. This point is even more critical in the particular case of beams. Indeed, beam consitutive laws involve the second derivative of the displacement and thus standard 2D FE cannot be used directly.

Non-Uniform Rational B-Spline functions (NURBS) are standards in Computer Aided Design, computer graphics and animation. They have the capability to be "p" enriched, i.e. increasing the polynomial order, while high continuity is maintained. These functions are thus extremely interesting for DIC. They grew out of the pioneering work of Pierre Bézier's development of Bézier curves and surfaces in the late 1960s. B-Spline and later NURBS and T-Splines were developed since then (see, e.g. Farin 
(1995); Piegl and Tiller (1997); Cohen et al (2001); Rogers (2001)). Despite a strong coupling between design and analysis in the global design of industrial products, such functions were not used in the analysis until recently. The work of Hughes et al (2005) on Isogeometric Analysis introduced NURBS functions in a "finite element" framework to represent both geometry and solution fields. Despite a real interest in the geometric representation build into isogeometric analysis, the smoothness of NURBS and B-Spline functions is the key ingredient that drove the use of the approach in various domains of analysis such as turbulence and fluid-structure interactions (Bazilevs et al 2007, 2008; Akkerman et al 2008); Cahn-Hilliard phase field modeling (Gomez et al 2008), incompressible problems (Auricchio et al 2007; Elguedj et al 2008) and structural dynamics (Hughes et al 2008).

In the present paper, we propose to explore the capability of NURBS functions to solve the DIC "compromise" with two examples: a compression test on a wood sample and a three-point bending test on a steel beam. After an introduction to the DIC formulation and the basis of NURBS, a methodology for error assessment is proposed and then applied to each of the two examples.

\section{Digital Image Correlation algorithm}

\subsection{Optical flow principle}

If successive images of the same sample are captured during its motion, a displacement $\mathbf{u}$ produces an advection of the local texture of the images. When analyzing a pair of images $f$ and $g$, the optical flow equation reads

$$
f(\mathbf{x})=g(\mathbf{x}-\mathbf{u}(\mathbf{x})) .
$$


The principle of DIC is to determine $\mathbf{u}$ as accurately as possible. The problem is illposed by nature and the first step is to try and solve it in "average", i.e. by minimizing a global error

$$
\eta^{2}=\iint_{\Omega} \Phi^{2}(\mathbf{x}) \mathrm{d} \mathbf{x}
$$

where $\Omega$ is the domain of interest and $\Phi(\mathbf{x})$ defines the local error:

$$
\Phi(\mathbf{x})=[f(\mathbf{x})-g(\mathbf{x}-\mathbf{u}(\mathbf{x}))] .
$$

The usual approach consists in maximizing a correlation coefficient (Sutton et al 1983, 1986, 2000) by searching for a piecewise constant, bilinear or other canonical form of displacement field. The image is thus subdivided in zones of interest that can overlap and the maximization is performed independently over each of these zones. Note that B-spline functions have already been used by Cheng et al (2002) in this context.

It has been shown in Besnard et al (2006) that prescribing displacement continuity over the entire domain of interest plays a regularizing role. Because of the ill-posedness of the problem, the "amount" of regularization is directly related to noise sensitivity and thus measurement uncertainty.

\subsection{Resolution}

We use in the following the global approach proposed in Besnard et al (2006). After a description basis

$$
\mathbf{u}(\mathbf{x})=\sum_{n \in \mathcal{N}} a_{n} \boldsymbol{\psi}_{n}(\mathbf{x})=[\boldsymbol{\Psi}(\mathbf{x})]\{\mathbf{U}\}
$$

is adopted for the displacement field, an iterative Newton procedure is initiated in order to minimize $\eta^{2}$ (Eq. (2)). A sequence of linear system is solved until convergence 
of the solution increment $\mathrm{d} \mathbf{U}$ is obtained:

$$
[\mathbf{M}]^{(i)}\{\mathrm{d} \mathbf{U}\}=\{\mathbf{b}\}^{(i)},
$$

with

$$
M_{n m}^{(i)}=\iint_{\Omega}\left(\boldsymbol{\psi}_{n} \cdot \nabla g\left(\mathbf{x}-[\mathbf{\Psi}]\{\mathbf{U}\}^{(i)}\right)\right)\left(\boldsymbol{\psi}_{m} \cdot \nabla g\left(\mathbf{x}-[\mathbf{\Psi}]\{\mathbf{U}\}^{(i)}\right)\right) \mathrm{d} \mathbf{x},
$$

and

$$
b_{n}^{(i)}=\iint_{\Omega}\left(\boldsymbol{\psi}_{n} \cdot \nabla g\left(\mathbf{x}-[\mathbf{\Psi}]\{\mathbf{U}\}^{(i)}\right)\right)\left(f(\mathbf{x})-g\left(\mathbf{x}-[\mathbf{\Psi}]\{\mathbf{U}\}^{(i)}\right)\right) \mathrm{d} \mathbf{x},
$$

$\nabla$ denoting spatial derivation and $i$ the current iteration. For a detailed description of the algorithm and its implementation the interested reader may refer to Réthoré et al (2007b)

\section{Non-Uniform Rational B-Splines and Isogeometric Analysis}

Non-Uniform Rational B-splines are a standard tool for describing and modeling curves and surfaces in computer aided design and computer graphics (see Piegl and Tiller (1997); Rogers (2001) for an extensive description of these functions and their properties). In this work, we use NURBS as a DIC/analysis tool, which is referred to as isogeometric analysis by Hughes et al (2005). The aim of this section is to present a brief overview of features and properties of NURBS-based isogeometric analysis for DIC problems.

3.1 B-spline and NURBS functions

B-splines are piecewise polynomial functions with a prescribed degree of continuity. Univariate B-spline basis functions are constructed from a knot vector, a set of coordinates in parametric space, $\Xi=\left\{\xi_{1}, \xi_{2}, \ldots, \xi_{n+p+1}\right\}$, where $\xi_{i} \in \mathbb{R}$ is the $i^{\text {th }}$ knot, 
$i$ is the knot index, $i=1,2, \ldots, n+p+1, p$ is the polynomial order, and $n$ is the number of basis functions. More than one knot can be placed at the same location in the parametric space.

B-spline basis functions for a given order $p$, are defined recursively in the parametric space by way of the knot vector $\Xi$. Beginning with piecewise constants $(p=0)$ we have

$$
N_{i, 0}(\xi)= \begin{cases}1 & \text { if } \xi_{i} \leq \xi<\xi_{i+1}, \\ 0 & \text { otherwise. }\end{cases}
$$

For $p=1,2,3, \ldots$, the basis is defined by the Cox-de Boor recursion formula:

$$
N_{i, p}(\xi)=\frac{\xi-\xi_{i}}{\xi_{i+p}-\xi_{i}} N_{i, p-1}(\xi)+\frac{\xi_{i+p+1}-\xi}{\xi_{i+p+1}-\xi_{i+1}} N_{i+1, p-1}(\xi)
$$

Note that the maximum continuity is $C^{p-1}$ when the first and last knots have unit multiplicity. The opposite configuration is when all the internal knot have $p$ multiplicity. In this case $p$-finite element functions $\left(C^{0}\right)$ are constructed ${ }^{1}$. Figure 1 shows $C^{0} \mathrm{FE}$ functions and $C^{p-1}$ NURBS functions for $p=2$ on a two-element mesh. A key point is that for a given polynomial order $p$, a description using NURBS functions have higher continuity and it also has fewer degrees of freedom: 4 instead of 5 in this case, $n_{e l}+p$ instead of $n_{e l} \times p+1$ in the general case $\left(n_{e l}\right.$ being the number of elements). Only these two cases will be considered in the following.

\section{A priori performance analysis}

The aim of this analysis is to evaluate a priori the performance of the correlation algorithm for a given basis function. As mentionned in the introduction, the choice of

1 Note that in standard FE analysis, Lagrange polynomials are used, which are different from the $C^{0}$ functions used here but produce similar results. 
the element size is a matter of compromise between correlation error and interpolation error. This is thus important to estimate the contribution of these two sources of error on the total error that will be obtained in a real case. We propose to proceed in three steps:

1. to evaluate the correlation error, we perform a DIC analysis over the domain of interest of the initial image with a deformed image which is artificially translated by $u_{t}$ pixel in each direction. As the prescribed displacement is constant no interpolation error arises at this step.

2. for a given displacement field $u_{s}$ that is not in the interpolation space of the basis function, we evaluate the interpolation error by comparing $u_{s}$ with its $L^{2}$ projection onto the basis function. As no DIC problem is solved, no correlation error arises at this step.

3. we perform a DIC analysis with an image that is obtained by artificially deforming the initial image with the displacement field of step 2 .

For step 1 , the value of $u_{t}=0.5$ pixel is chosen because it produces the maximum correlation error. The displacement prescribed at steps 2 and 3 is chosen of the following form:

$$
u_{s}(s)=u_{0} \sin \left[\frac{\omega}{2 \pi}\left(1-\left|2 \frac{s}{\max (s)}-1\right|\right)^{2}\right] \text {, }
$$

where $\omega=n_{\omega} \pi^{2}, u_{0}=1$ pixel and $s$ the co-ordinate of the current pixel in a certain frame. Whereas the amplitude of the displacement is constant, the amplitude of its derivatives is varying. Further the sine function is not in the interpolation space of any polynomial basis.

For step 2, the $L^{2}$ projection is performed in the following way:

$$
\operatorname{Arg}\left[\min \iint_{\Omega}\left(u-u_{s}\right)^{2} \mathrm{~d} \mathbf{x}\right] .
$$


For each step, the error is obtained by taking the standard deviation of the gap between the computed displacement (by DIC or $L^{2}$ projection) and the prescribed displacement ( $u_{t}$ or $\left.u_{s}\right)$, i.e. the RMS (root mean square) error.

Comparing the results of step 3 with those of steps 1 and 2, we can thus evaluate the competition between correlation error and interpolation error when an actual DIC analysis is performed. The analysis is then carried out varying the element size $h$, the polynomial degree $p$ and the level of continuity $\left(C^{0}\right.$ or $\left.C^{p-1}\right)$ to estimate the influence of these parameters on the performance of DIC.

\section{2D DIC analysis of a compression test on a wood sample}

\subsection{Test description}

The goal of this test is to investigate stiffness variations in the radial direction for a specimen of spruce (softwood). In the transverse plane, wood is constituted by annual rings. In the specimen presented here, the width of the rings is around $1 \mathrm{~mm}$. Each growth can be divided between early wood, brighter on the photograph (Figure 2a), and late wood, darker.

The growth direction goes from the top left of the photograph to the bottom right. Early wood grows rapidly during spring and is very light $\left(0.2\right.$ to $\left.0.3 \mathrm{~g} / \mathrm{cm}^{3}\right)$. Its density varies continuously from the beginning of early wood to the end of late wood, where density can reach $1.2 \mathrm{~g} / \mathrm{cm}^{3}$. This density variation leads to important stiffness variation across the annual ring: for example shear modulus goes from 15 to $300 \mathrm{MPa}$. As a consequence, in such a compression test, strains decrease continuously across the growth ring and then increase deeply between one year late wood and the following year early wood. This rapid transition is uneasy to catch with classical DIC techniques. 
The initial image and the domain of interest is shown by Figure 2a. The domain of interest is a square of 640 -pixel side. Figure $2 \mathrm{~b}$ shows the deformed image. The right side of the specimen has its horizontal displacement fixed and the compressive load is applied on the left side. The vertical displacement along the left and right faces is not fixed.

5.2 Uncertainty analysis

For the uncertainty analysis, the frequency parameter of $u_{s}$ is $n_{\omega}=5$. The co-ordinate $s$ is set to the sum of the $x$ and $y$ co-ordinates so that the gradient of $u_{s}$ is along the first diagonal of the domain of interest. For the analysis, the initial image is deformed along the $x$ and the $y$ direction with the same prescribed displacement $u_{s}$ (all the component of the strain tensor $\varepsilon_{x x}, \varepsilon_{y y}$ and $\varepsilon_{x y}$ thus have the same value at a given pixel). Figure 3a shows this displacement field. One would have noticed that the wood structure gives prefered orientation to the image gradient. Yet, from Eq. (1), the displacement are measurable in the direction of the image gradient only. For the uncertainty analysis, the artificial displacement field $u_{s}$ is more or less parallel to the wood line structure, i.e. orthogonal to the image gradient. The setup is thus the least favorable. The element size $h$ is successively set to $16,32,64$ and 128 pixels. $C^{p-1}$ NURBS functions and $C^{0} \mathrm{FE}$ functions of degree 1 to 3 are tested.

As an illustration, Figure 3 presents the evolution of the displacement $u_{x}$ and the strain $\varepsilon_{x x}$ along the diagonal of the domain of interest. For 32-pixel elements with $C^{p-1}$ NURBS functions with $p=3, u_{x}$ and $\varepsilon_{x x}$ obtained by DIC and by $L^{2}$ projection are plotted and compared with the prescribed displacement and strain. With this set 
of parameters, no visible difference is observed for the displacement whereas small deviation of the correlated strain is obtained at the maximum amplitude points.

On Figure 4 the correlation error (step 1) and the interpolation error (step 2) for displacement and strains are plotted as a function of the mesh size for $C^{0} \mathrm{FE}$ functions and $C^{p-1}$ NURBS functions of different degrees. One observes that for displacement or strain the correlation error decreases as a power law of $h$ whereas the interpolation error increases as a power law of $h$. For all the cases (whatever the value of $p$ and the continuity) the exponant of the correlation error is about -1.5 for the displacement (Figure 4a) and about -2.5 for strains (Figure 4c). This difference could have been anticipated from a dimensional analysis. For $C^{p-1}$ NURBS functions the correlation error is almost independent on the degree $p$ whereas for $C^{0} \mathrm{FE}$ functions it is increased by a factor of 3 between $p=1$ and $p=2$ and by a factor of 2 between $p=2$ and $p=3$ for the displacement. For strains the same observations holds but with a factor of 6 between $p=1$ and $p=2$ and a factor of 3 between $p=2$ and $p=3$. Concerning the interpolation error, whatever the continuity, power law exponants of 2,3 , and 4 for $p$ from 1 to 3 are obtained for the displacement and 1, 2, and 3 for strains. The only significative difference between $C^{p-1}$ NURBS functions and $C^{0} \mathrm{FE}$ functions is revealed for $p=3$ with lower error level on displacement and strains for the latter.

At this point, one of the main advantages of using $C^{p-1}$ NURBS functions is clearly pointed out: increasing the polynomial order $p$ allows for higher convergence rate of the interpolation error but the correlation error remains constant. Indeed, for $C^{p-1}$ NURBS functions increasing $p$ by 1 costs only 1 degree of freedom per dimension whereas it $\operatorname{costs} n_{e l}$ degrees of freedom per dimension for $C^{0} \mathrm{FE}$ functions. Further, increasing the order of continuity not only decreases the number of degrees of freedom but also increases the size of the support of each NURBS function. Hence, for $C^{p-1}$ NURBS 
functions, increasing $p$ only raises by a very small "amount" the under-determination of the system and the correlation error is unchanged.

Figure 5 allows to compare the total error (step 3$)$ for bilinear $\mathrm{FE}(p=1), C^{0} \mathrm{FE}$ with $p=3$ and $C^{p-1}$ NURBS with $p=3$. For $p=1$, (Figure 5 a and Figure $5 \mathrm{~d}$ ), the total error is controled by the interpolation error and lowest error level is obtained for 16-pixel elements: $1.110^{-2}$ pixel for displacement and $0.810^{-3}$ for strains. On the contrary, for $C^{0} \mathrm{FE}$ functions with $p=3$ (Figure $5 \mathrm{~b}$ and Figure 5e), the correlation error overcomes the interpolation error for displacement and strains. The lowest error level is obtained for 64-pixel elements: $1.110^{-2}$ pixel for displacement and $0.410^{-3}$ for strains. For $C^{p-1}$ NURBS function with $p=3$, the competition between correlation error and interpolation error is well balanced and an optimal choice of the element size appears to be 32 pixels. For this element size the global error is $1.010^{-2}$ pixel for displacement and $0.410^{-3}$ for strains. One may also consider the intersection point between the line of the correlation error and the one of the interpolation error. This point defines the minimum total error that is potentially reachable with the basis function. For bilinear FE, we have $4.010^{-3}$ pixel and $6.010^{-4}$ for displacement and strains, for $C^{0} \mathrm{FE}$ with $p=3,210^{-3}$ pixel and $1.510^{-4}$ and for $C^{p-1}$ NURBS with $p=3,0.910^{-3}$ pixel and $0.510^{-4}$. This shows that the improvement brought by the use of NURBS is even higher.

\subsection{Results}

Now that the performance of the correlation algorithm has been evaluated, the analysis is performed between the initial image (Figure 2a) and the final one (Figure 2b). A first analysis is performed using 32-pixel FE and a second one using 64-pixel element 
based $C^{p-1}$ NURBS functions with $p=3$. The deformed meshes and the correlation residual are presented in Figure 6 while Figure 7 shows three components of the strain tensor $\left(\varepsilon_{x x}, \varepsilon_{y y}\right.$ and $\varepsilon_{n t}$ the shear strain in the co-ordinate system aligned with the wood structure).

The displacement magnitude is up to 50 pixels and localized deformation patterns are obtained due to the density variation of wood. Using 32-pixel FE or 64-pixel $C^{p-1}$ NURBS functions with $p=3$, the deformed look similar and no difference is visible on the residual map: the mean residual is $1.36 \%$ of the image dynamic for FE and $1.43 \%$ for NURBS. On the contrary, significant differences between the two analysis are obtained in Figure 7. Figures $7 \mathrm{a}, \mathrm{b}, \mathrm{d}$,e show the strains $\varepsilon_{x x}$ and $\varepsilon_{y y}$ in the image co-ordinate system that is aligned with the mesh direction. FE leads to piecewise constant strains with discontinuities across element edges. Further, the strain patterns are aligned with the mesh. Using $C^{p-1}$ NURBS functions with $p=3$ and a coarser mesh, the variation of strains between early wood and late wood is better captured. This is most significant in Figures $7 \mathrm{c}, \mathrm{f}$ where the map of $\varepsilon_{n t}$ (the shear strain in a co-ordinate system aligned with the wood structure) is shown. It is observed that the strain is concentrated within the early wood (up to $5 \%$ strain) whereas the late wood remains almost undeformed. Using FE, this transition is also captured but its geometrical support is "mesh dependent" as the iso-strain contour are aligned with the mesh. In addition to a better compromise between correlation error and interpolation error, another advantage of using NURBS functions for DIC is thus emphasized: the strain pattern are described with more accuracy and without "mesh dependent" geometrical structures. 


\section{Beam DIC analysis of a three-point bending test}

6.1 Test description

The use of NURBS for DIC is now tested on a three-point bending test, Figure 8. The set-up is fixed into a servo-hydraulic tension-compression machine of $10 \mathrm{kN}$ capacities. The specimen is a steel beam of a 10x10 mm section rested on 2 cylinders with a 120 mm span. During the loading, a serie of 213 images are taken with a digital camera (2048x2048 pixels). With this configuration, 1 pixel is equal to $0.066 \mathrm{~mm}$. Figure 8 shows 4 stages of the loading : a) is the undeformed state (step 0), b) is a deformed image in the elastic regime (step 60), c) is an image at the maximum load (step 200) and d) is the final image after unloading (step 213). The domain of interest covers $140 \times 1800$ pixels centered below the central point.

\subsection{Beam kinematics}

For this test on a steel beam, the identification procedure should focus on the relationship between the flexural moment and the curvature. The kinematic quantity to extract from the measurement is thus the cuvature field along the beam axis. One can try a $2 \mathrm{D}$ approach using $\mathrm{FE}$ and then average the displacement over the beam width but this will not give access to the curvature as second order derivatives of FE shape functions vanish.

The global formulation of DIC we use herein allows for decomposing the displacement on arbitrary basis function. It is thus possible to introduce a basis function constructed according to beam kinematics as in Hild et al (2009). We start with functions that capture the variation of the displacement $u_{x}^{b}$ orthogonal to the beam axis $\boldsymbol{y}$ 
due to bending:

$$
u_{x}^{b}(y)=\sum_{n \in \mathcal{N}} u_{n}^{b} \psi_{n}(y)
$$

The displacement $u_{y}^{b}$ along the beam axis is derived from the rotation of the section. Using Euler-Bernoulli assumptions, the rotation $\theta^{E B}$ is equal to the derivative of $u_{x}^{b}$. It thus writes:

$$
\theta^{E B}(y)=\sum_{n \in \mathcal{N}} u_{n}^{b} \psi_{n}^{\prime}(y)
$$

where $\psi_{n}^{\prime}=\frac{\mathrm{d} \psi_{n}}{\mathrm{~d} y}$ and the unknown $u_{n}^{b}$ are the same as those describing the evolution of $u_{x}^{b}$. For large rotations or when the length of the beam is of the same order of magnitude as its width, Timoshenko assumptions are more appropriate. In this case, the rotation $\theta^{T}$ is independent from the orthogonal displacement and its writting involves its own degrees of freedom:

$$
\theta^{T}(y)=\sum_{n \in \mathcal{N}} t_{n} \psi_{n}^{\prime}(y)
$$

Note the relation between displacement and rotation is invalidated but we keep describing the rotation with the derivatives $\psi_{n}^{\prime}$ of the functions that describe the displacement. The curvature is directly obtained by differenciation of the rotation whatever the kinematic assumption. The axial displacement $u_{y}$ is now written in term of a generic rotation field $\theta$ which will match $\theta^{E B}$ or $\theta^{T}$ :

$$
u_{y}^{b}(x, y)=-x \quad \theta(y)
$$

To accomodate test imperfection, a traction-compression kinematic is added to this bending description. A translation and a constant strain define this additional motion for the entire domain of interest of length $\ell$. Two unknowns $\left(u_{1}^{t}\right.$ and $\left.u_{2}^{t}\right)$ are thus required to decompose this displacement $u_{y}^{t}$ :

$$
u_{y}^{t}=u_{1}^{t}+\frac{y}{\ell} u_{2}^{t} .
$$


In the following $\psi_{n}$ functions are chosen as $C^{0} \mathrm{FE}$ functions or $C^{p-1}$ NURBS functions. Here the advantage of NURBS functions is straightforward as they ensure the continuity of the rotation and curvature along the axis (for $p \geq 3$ ). Due to the crude description of the tension kinematic, NURBS functions also warrant the continuity of the axial strain. Note that functions of degree at least 2 must be used for non-zero curvature fields.

\subsection{Uncertainty analysis}

The same methodology as for the previous example is adopted in this section. The frequeny parameter of the prescribed displacement $u_{s}$ is $n_{\omega}=5$. The co-ordinate $s$ is $y$ and $u_{s}$ is affected to the orthogonal displacement $u_{x}$. Euler-Bernoulli's assumptions are then invoked to derive the corresponding rotation, axial displacement and curvature. The analysis is carried out for $h$ being equal to 8, 18, 25, 60, 100 and 225 pixels in the axial direction, the size of the beam elements in the orthogonal direction being the width of the domain of interest. $C^{p-1}$ NURBS functions and $C^{0}$ FE functions of degree 2 to 5 are considered.

Figure 9 shows the comparison of the displacement, rotation and curvature between the prescribed displacement, the projected displacement and the measured displacement for $h=100$ pixels and $C^{p-1}$ NURBS functions with $p=5$. Except at the center of the beam where the highest curvature amplitude is reached, a quasi-perfect agreement is obtained. Concerning the correlation error and the interpolation error, the results and their interpretation are similar to those for the 2D case. For the sake of clarity, only the results for NURBS function with $p=5$ are presented in Figure 10. Power law decrease, respectively increase, is obtained for the correlation error, respectively the 
interpolation error. Concerning the correlation error, the exponent for the displacement is 0 and is decremented by -1 each time this field is differenciated: we have -1 for the rotation and -2 for the curvature. The behaviour of the interpolation error is not as clear but it goes from an exponent that should be 2 for the displacement to 4 for the curvature. The total error is limited by its two contributions (correlation error and interpolation error). For the displacement, a minimum of $4.010^{-3}$ pixel is reached for $h=8$ pixels. The minimum of the total error at $6.010^{-5}$ on the rotation is obtained for $h=60$ pixels. The total error on the curvature fits with a strong accordance the maximum between the correlation error and the interpolation error; the minimum value of $1.510^{-6}$ pixel $^{-1}$ is obtained for $h=60$ pixels.

Figure 11 compares the correlation error for three discretizations : $C^{0} \mathrm{FE}$ functions with $p=5$ and Euler-Bernoulli assumptions, $C^{p-1}$ NURBS functions with $p=5$ and Euler-Bernoulli assumptions and $C^{p-1}$ NURBS functions with $p=5$ and Timoshenko assumptions. The evolution of the error on the rotation and the curvature are presented. Despite the additional number of unknown invoked in the Timoshenko model to describe the rotation, no significant difference is obtained compared to the EulerBernoulli type beam. On the contrary, $C^{0} \mathrm{FE}$ functions give a raise of the correlation error by a factor of roughly 10. It is thus demonstrated that the higher continuity order provided by NURBS functions allows for much more robust measurements of rotation and curvature fields.

\subsection{Results}

The series of deformed images presented in Figure 8 are now analyzed. A 2D analysis using 16-pixel elements with bilinear FE functions is considered as a reference because 
this description has no kinematic assumption. Figure 12 compares the deformed shape for the three deformed images obtained with the reference analysis and one performed with 10 elements using $C^{p-1}$ NURBS functions with $p=5$ and Euler-Bernoulli assumptions. A very good agreement is obtained between these analysis for the three stages. A maximum displacement of 40 pixels is obtained at the maximum load level that leads to an important plastic deformation of the beam. The remaining displacement after unloading is about 20 pixels. Figure 13 shows the evolution of the rotation and curvature for the second analysis. The rotation vanishes at the centre of the beam whatever the applied load and symmetric evolutions are obtained. For the first loading step (step 60 ), the maximum rotation is \pm 0.017 at both ends of domain of interest. At step 200, the maximum rotation is \pm 0.058 but it seems that higher gradients are measured near the centre of the beam. For step 213, the rotation remains constant \pm 0.022 appart from the region near the center of the beam where strong plastic strains occurred. These results are enforced by examining the evolution of the curvature (Figure 13b). At step 60, a piecewise linear curvature is obtained which confirms the elastic state at this step. For step 200, a strong intensification of the curvture is obtained in a 500-pixel central section of the beam with curvature as high as $-2.610^{-4}$ pixel $^{-1}$. In the rest of the beam the evolution of the curvature remains linear which shows an elastic behaviour. After unloading, the curvature vanishes out of the central section of the beam wherein remaining curvature with a maximum level of $-1.810^{-4}$ pixel $^{-1}$ is measured.

For comparison purposes, Figure 14a,c show the rotation at step 200 for 4 analysis: the reference analysis with 2D FE (the displacement has been averaged over the beam width and then differenciated to obtain the rotation), 10 Euler-Benoulli beam elements using $C^{0}$ FE functions with $p=5$ and $C^{p-1}$ NURBS functions with $p=5$ and 
10 Timoshenko beam elements using $C^{p-1}$ NURBS functions with $p=5$. All the results are in very good agreement, which is also confirmed by the error maps shown in Figure 15, mean correlation residual being respectively $1.38 \%, 1.43 \%, 1.43 \%$ and $1.40 \%$ of the image dynamic. Only a slight difference in the rotation between the last two analysis (Euler-Benoulli and Timoshenko) is detected. The differenciation that is performed to obtain the curvature, presented in Figure 14b,d, enlarges the differences between these analysis. Of course, the curvature cannot be obtained from the $2 \mathrm{D}$ analysis despite this is the meaningfull data for identification. FE and NURBS curvatures follow the same evolution except that the FE curvature field is discontinuous which will not be appropriate for consitutive law identification. For this step, where quite large and concentrated rotations are obtained, it appears that the Euler-Bernoulli beam model underestimates the maximum level of curvature of about $0.310^{-4} \mathrm{pixel}^{-1}$ which is much higher than the uncertainty of $0.0510^{-4}$ pixel $^{-1}$ for the corresponding element size. Note that the uncertainty analysis has been carried out with curvature levels similar to those of the experiment (about $2.010^{-4}$ pixel $^{-1}$ ).This again increases the confidence in the experimental results.

Last, Figure 16 presents the axial strain $\varepsilon_{y y}$ in percent for the four above compared analysis at the maximum load level. In addition to the strain level, the iso-0 line (the neutral axis of the beam) of $\varepsilon_{y y}$ is drawn with a black line. For the $2 \mathrm{D}$ reference analysis, Figure 16a, the strain map is noisy and many iso-0 lines exist which makes the determination of the neutral axis quite uneasy. For $C^{p-1}$ NURBS functions, EulerBernoulli and Timoshenko beam kinematics, Figure 16b,d, provide comparable results with a maximum amplitude of \pm 0.02 of $\varepsilon_{y y}$ in the middle section of the domain of interest. Note that the neutral axis is not located on the axis of the beam as it is the case for elastic states. At this step when most of the deformation is concentrated in the 
middle of the beam, the neutral axis appears as a curved line that goes from the tension side 600 pixels bellow the middle of the beam to the tension side 600 pixels above the middle. This line goes through the centre of the beam section at the middle of the beam which suggests that the material has kept a tension / compression symmetry. For $C^{0}$ FE functions, the continuity of the axial strain is not ensured and an erratic description of the neutral axis is obtained.

\section{Conclusions}

In this paper, we analysed the benefit of using high continuity NURBS functions for displacement measurement by Digital Image Correlation. After a brief remainder of the problem formulation and the NURBS definition and properties, we proposed a methodology to assess how given basis functions may solve the DIC compromise. Indeed, through the two examples we proposed further, the competition between the correlation error (due to the illposedness of the problem) and the interpolation error (due to the variability of the displacement field to capture) was illustrated. Further, when the displacement is aimed at feeding non-linear consitutive law identification, non-homogeneous strain states are expected. The interpolation error must then be reduced. In a finite element context this means decreasing the mesh size or increasing the degree of the shape functions. We showed that this leads to a larger number of degrees of freedom and thus higher uncertainty and noise levels. Using NURBS functions, that can be seen as high degree FE with high continuity $\left(C^{p-1}\right.$ for degree $\left.p\right)$, the number of degrees of freedom is decreased and the support of each function is enlarged. The conditioning of the problem is thus significantly improved and uncertainty and noise levels significantly reduced. For the $2 \mathrm{D}$ example as well as for the one using beam kine- 
matics, NURBS functions showed their ability to capture from digital images not only a displacement field but also its first (and second) derivatives with lower uncertainty levels.

\section{References}

Akkerman I, Bazilevs Y, Calo V, Hughes T, Hulshoff S (2008) The role of continuity in residualbased variational multiscale modeling of turbulence. Computational Mechanics 41(3):371378

Auricchio F, da Veiga LB, Buffa A, Lovadina C, Reali A, Sangalli G (2007) A fully locking-free isogeometric approach for plane linear elasticity problems: a stram function formulation. Computer Methods in Applied Mechanics and Engineering 197:160-172

Bazilevs Y, Calo V, Cottrell J, Hughes T, Reali A, Scovazzi G (2007) Variational multiscale residual-based turbulence modeling for large eddy simulation of incompressible flows. Computer Methods in Applied Mechanics and Engineering 197(1-4):173-201

Bazilevs Y, Calo V, Hughes T, Zhang Y (2008) Isogeometric fluid-structure interaction: theory, algorithms and computations. Computational Mechanics 43(1):3-37

Besnard G, Hild F, Roux S (2006) 'finite-element' displacement fields analysis from digital images: Application to portevin-le châtelier bands. Experimental Mechanics 46(6):789803

Cheng P, Sutton MA, Schreier HW, McNeill SR (2002) Full-field speckle pattern image correlation with b-spline deformation function. Experimental Mechanics 42(3):344-352

Cohen E, Riesenfeld R, Elber G (2001) Geometric Modeling with Splines: An Introduction. A.K. Peters Ltd., Wellesley, Massachusetts

Elguedj T, Bazilevs Y, Calo V, Hughes T (2008) B-bar and F-bar projection methods for nearly incompressible linear and non-linear elasticity and plasticity based on higher-order NURBS elements. Computer Methods in Applied Mechanics and Engineering 197:2732-2762

Farin G (1995) NURBS Curves and Surfaces: from Projective Geometry to Practical Use. A.K.

Peters Ltd., Natick, MA 
Gomez H, Calo V, Bazilevs Y, Hughes T (2008) Isogeometric analysis of the Cahn-Hilliard phase field model. Computer Methods in Applied Mechanics and Engineering 197:43334352

Hild F, Roux S, Gras R, Guerrero N, Marante M, Florez-Lopez J (2009) Displacement measurement technique for beam kinematics. Optics and Lasers in Engineering 47(3-4):495-503

Hughes T, Cottrell J, Bazilevs Y (2005) Isogeometric analysis: CAD, finite elements, NURBS, exact geometry and mesh refinement. Computer Methods in Applied Mechanics and Engineering 194:4135-4195

Hughes T, Reali A, Sangalli G (2008) Duality and unified analysis of discrete approximations in structural dynamics and wave propagation: Comparison of p-method finite elements with k-method NURBS. Computer Methods in Applied Mechanics and Engineering 197(49$50): 4104-4124$

Piegl L, Tiller W (1997) The NURBS Book (Monographs in Visual Communication), 2nd edn. Springer-Verlag, New York

Réthoré J, Hild F, Roux S (2007a) Extended digital image correlation with crack shape optimization. International Journal for Numerical Methods in Engineering 73(2):248-272, DOI $10.1002 /$ nme.2070

Réthoré J, Hild F, Roux S (2007b) Shear-band capturing using a multiscale extended digital image correlation technique. Computer Methods in Applied Mechanics and Engineering 196(49-52):5016-5030, DOI 10.1016/j.cma.2007.06.019

Rogers D (2001) An Introduction to NURBS With Historical Perspective. Academic Press, San Diego, CA

Roux S, Hild F (2006) Stress intensity factor measurement from digital image correlation: postprocessing and integrated approaches. International Journal of Fracture 140(1-4):141-157 Sutton M, Wolters W, Peters W, Ranson W, McNeill S (1983) Determination of displacements using an improved digital correlation method. Image Vision Computing 1(3):133-139

Sutton M, Cheng M, Peters W, Chao Y, McNeill S (1986) Application of an optimized digital image correlation method to planar deformation analysis. Image Vision Computing $4(3): 143-150$ 
Sutton M, McNeill S, Helm J, Chao Y (2000) Photomechanics, Springer, chap Advances in two-dimensional and three-dimensional computer vision, pp 323-372 


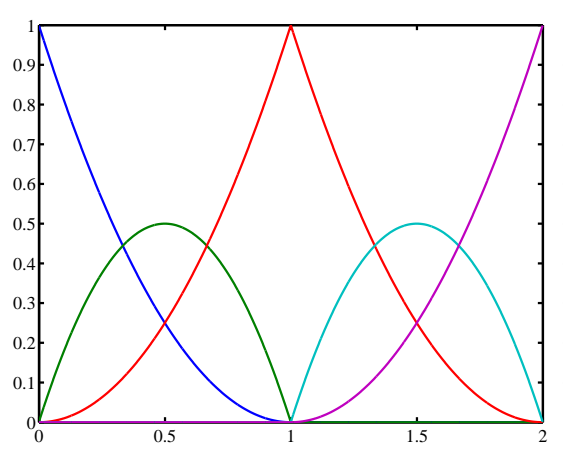

(a)

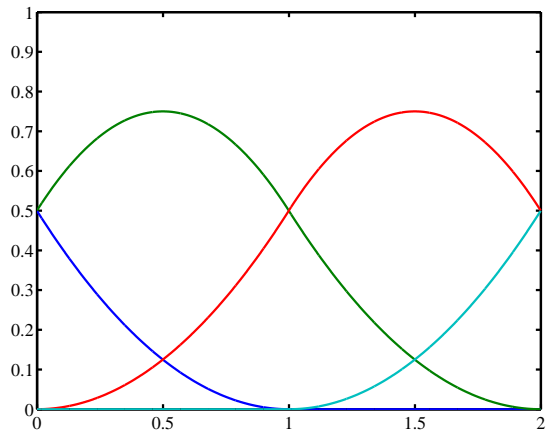

(b)

Fig. 1 (a) $p=2 C^{0}$ finite element functions and (b) $p=2 C^{p-1}$ NURBS functions for two elements 


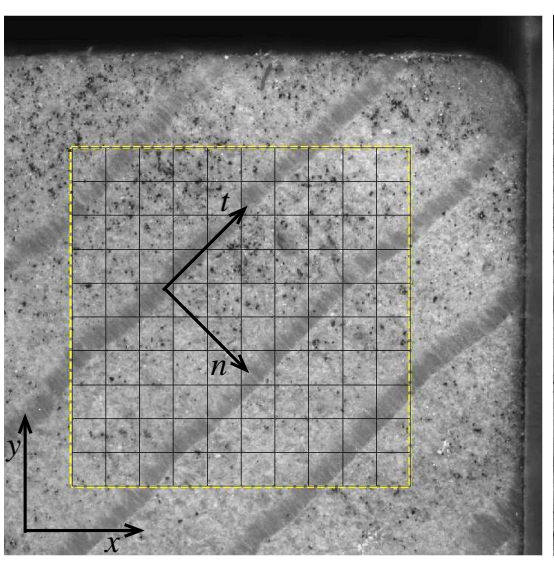

(a)

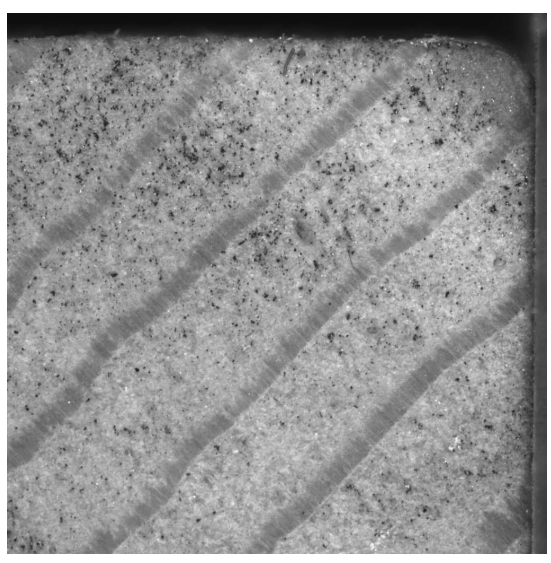

(b)

Fig. 2 Domain of interest for the wood sample on the initial image (a), the final state (b).

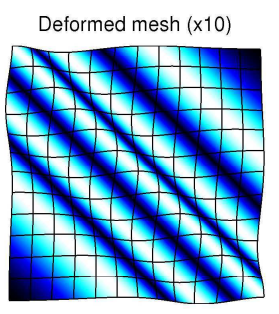

(a)

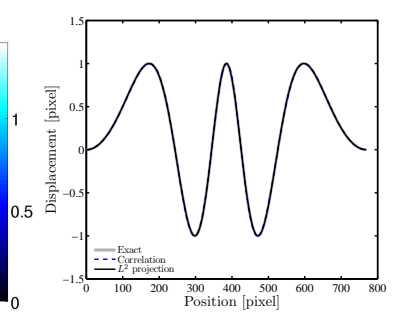

(b)

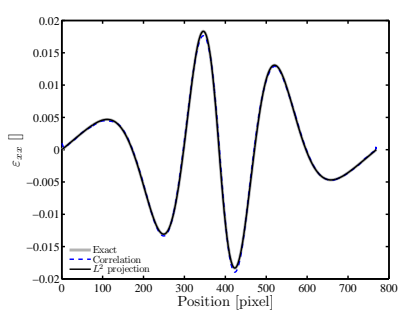

(c)

Fig. 3 Uncertainty study: the mesh for 64 -pixel elements deformed by the displacement field used for the uncertainty analysis (a)( the colormap gives the displacement norm in pixel) comparison of the displacement (b) and $\varepsilon_{x x}$ strain (c) along the diagonal of the domain of interest for 32-pixel elements with $C^{p-1}$ NURBS functions with $p=3$. 


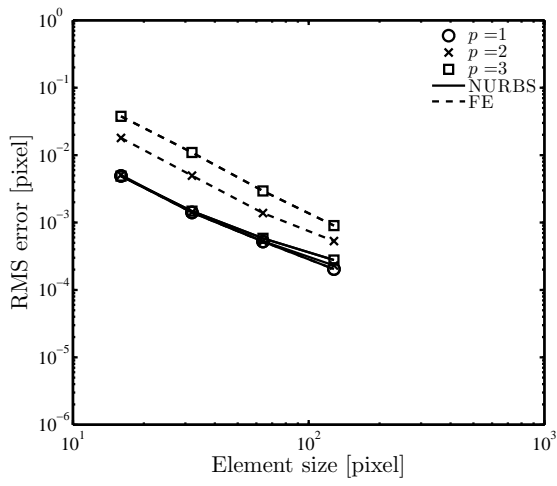

(a)

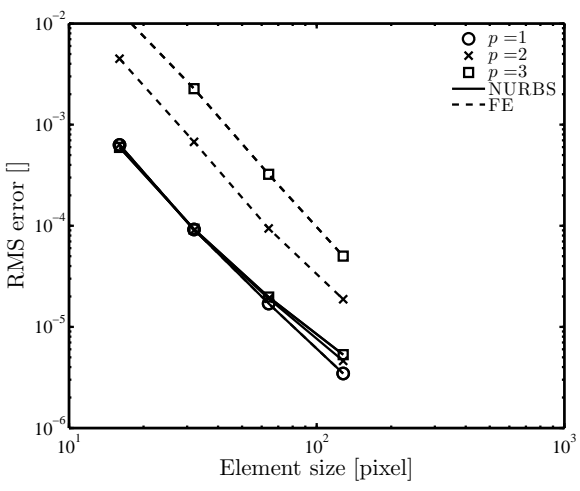

(c)

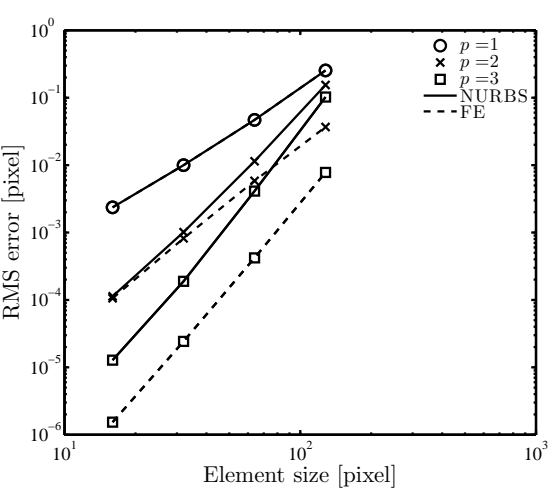

(b)

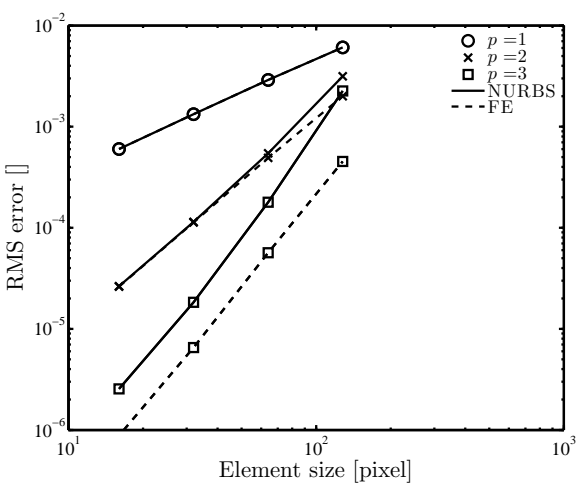

(d)

Fig. 4 Evolution of the correlation error (left) and interpolation error (right) as functions of the element size. Figure $(\mathrm{a}, \mathrm{b})$ shows displacement error and figures $(\mathrm{c}, \mathrm{d})$ strain error. 


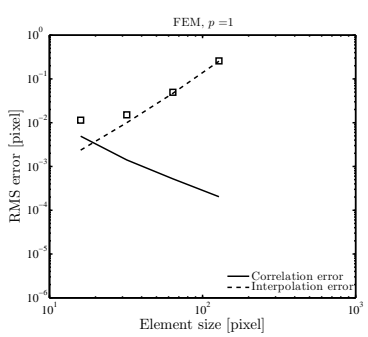

(a)

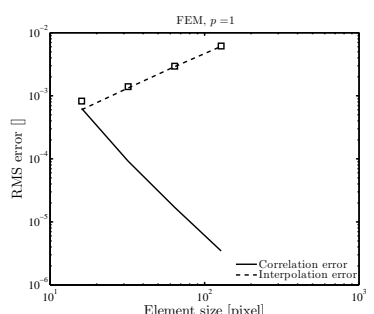

(d)

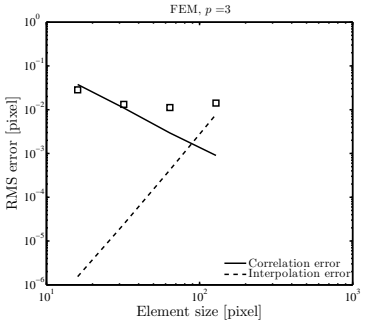

(b)

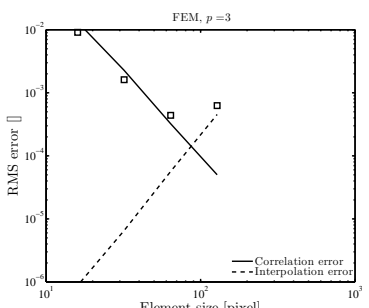

(e)

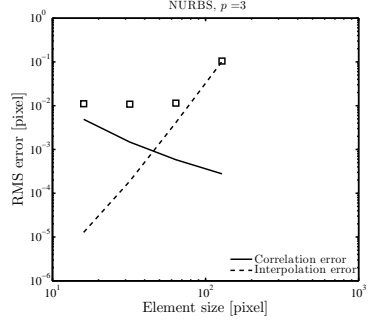

(c)

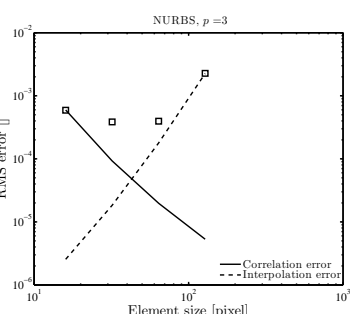

(f)

Fig. 5 Measurement uncertainty on displacement (top) and strain (bottom). From left to right bilinear FEM, bicubic FEM $\left(C^{0}, p=3\right)$ and NURBS $\left(C^{p-1}, p=3\right)$. The total error is depicted with square marks, the correlation error with lines and the interpolation error with dashed lines. 


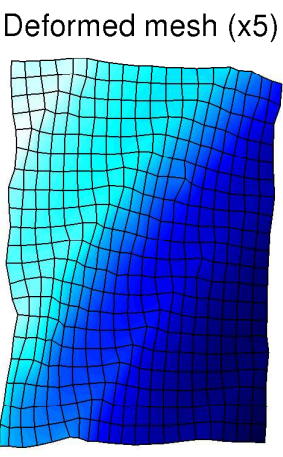

(a)

Deformed mesh (x5)

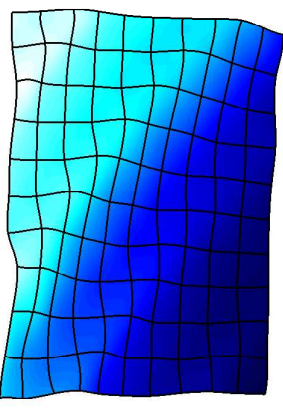

(c)

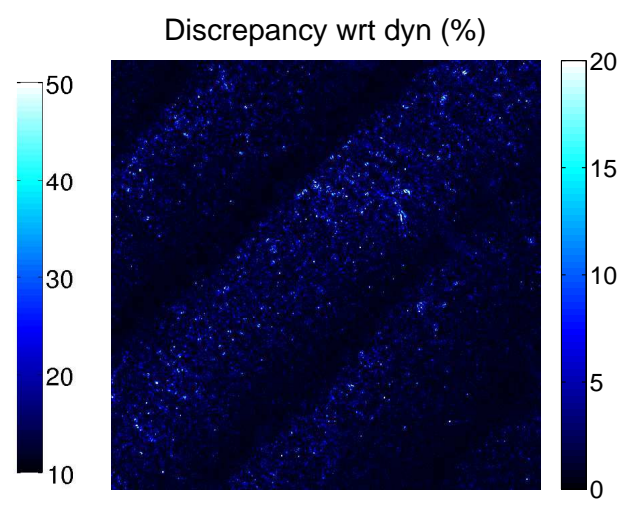

(b)

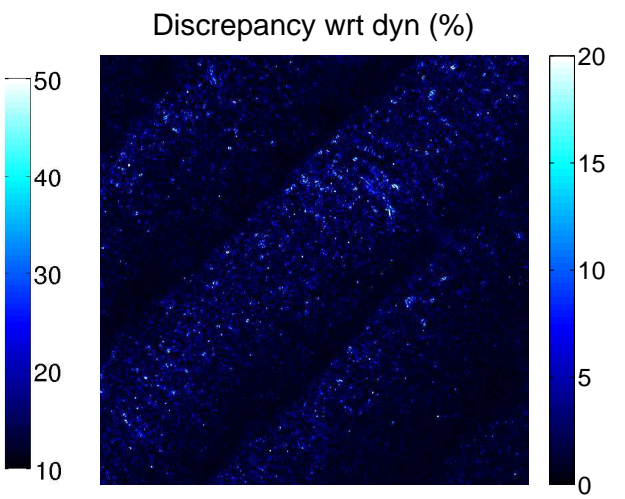

(d)

Fig. 6 Deformed mesh (left, amplification factor 10 in pixel) and error field (right) normalized by the dynamic of the initial image for FEM bilinear functions based on 32-pixel elements (top), NURBS functions with $p=3$ based on 64-pixel elements (bottom). 


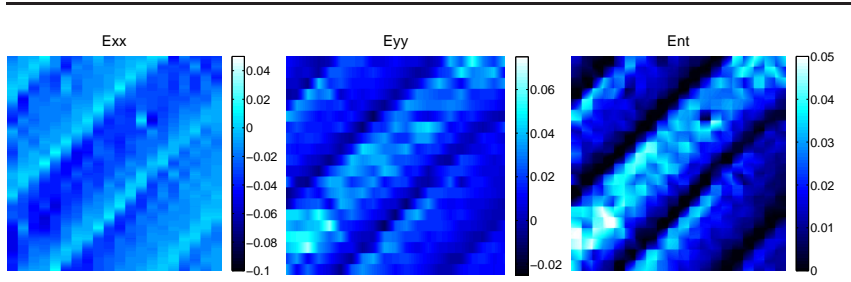

(a)

(b)

(c)

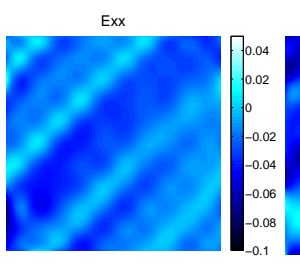

(d)

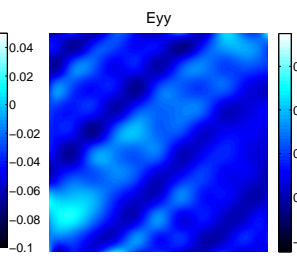

(e)

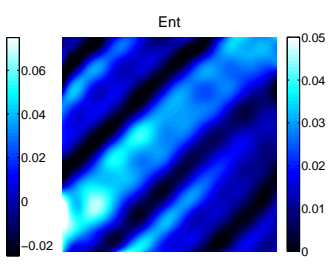

(f)

Fig. $7 \varepsilon_{x x}, \varepsilon_{y y}$ and $\varepsilon_{n t}$ strain for FEM bilinear functions based on 32-pixel elements (top), NURBS functions with $p=3$ based on 64-pixel elements (bottom). 


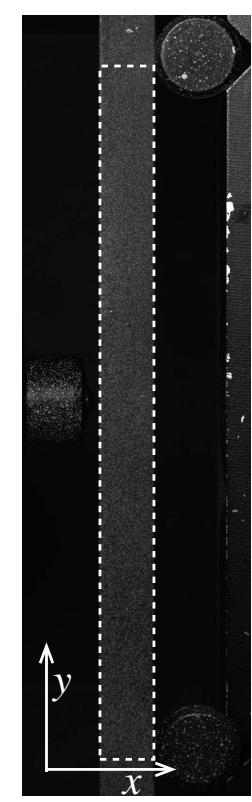

(a)

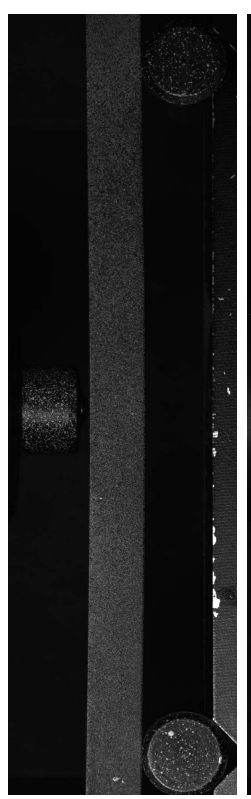

(b)

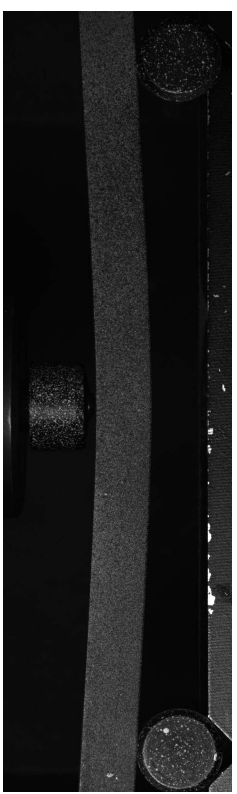

(c)

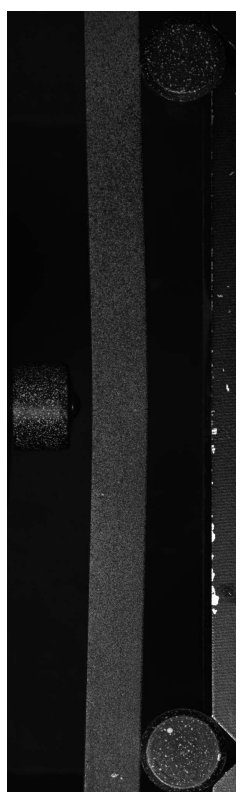

(d)

Fig. 8 Domain of interest for the steel beam on the initial image (a), deformed images: in the elastic regime (b), at the maximum load level (c) after unloading (d). 


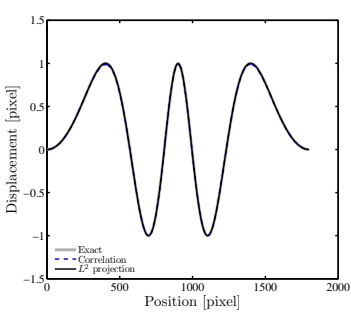

(a)

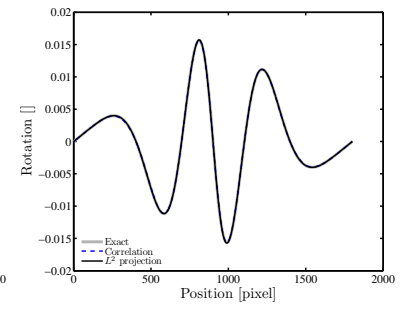

(b)

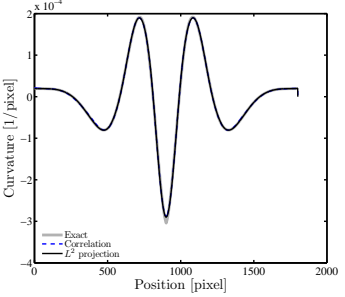

(c)

Fig. 9 Uncertainty study: comparison of the displacement (a) the rotation (b) and the curvature (c) along the beam axis for 100-pixel elements using $C^{p-1}$ NURBS functions with $p=5$. 


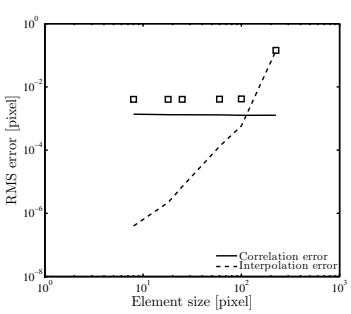

(a)

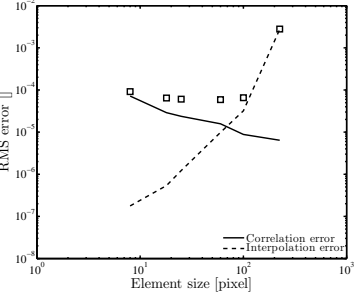

(b)

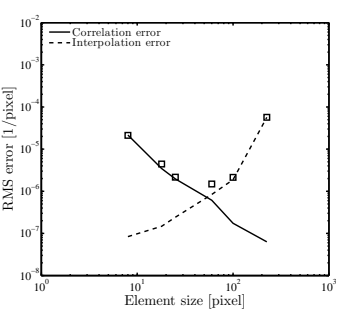

(c)

Fig. 10 For $C^{p-1}$ NURBS functions with $p=5$, evolution of the displacement uncertainty

(a), the rotation uncertainty (b) and the curvature uncertainty (c). 


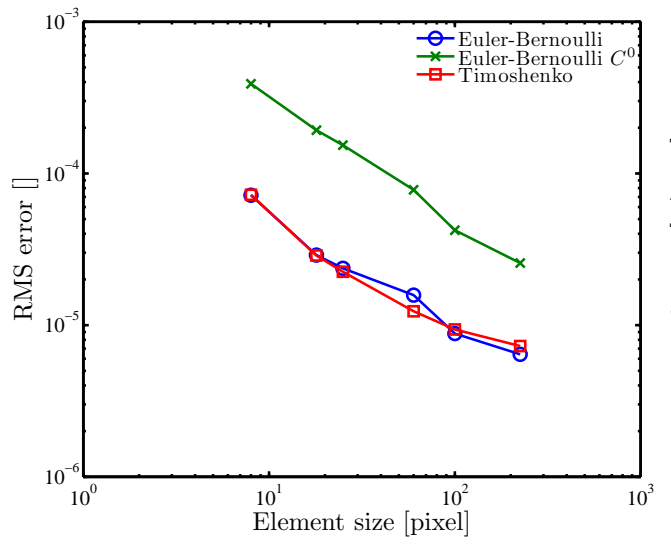

(a)

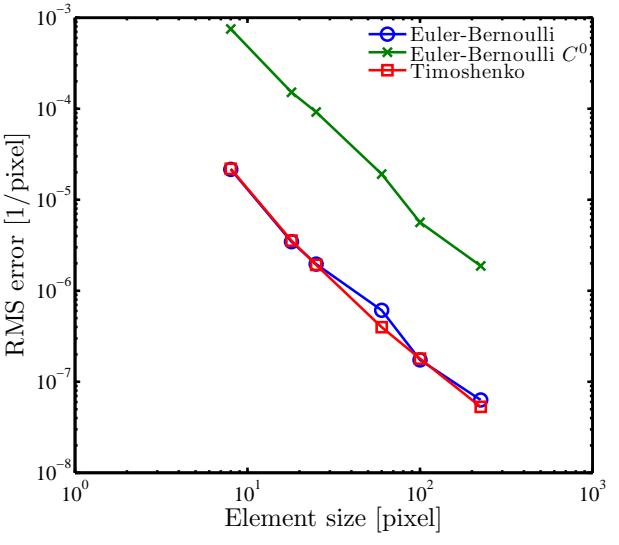

(b)

Fig. 11 Comparison of the correlation error on the rotation (a) and the curvature (b) for different discretization strategies. 


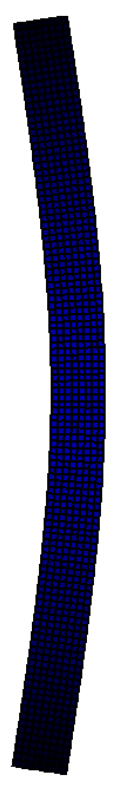

(a)

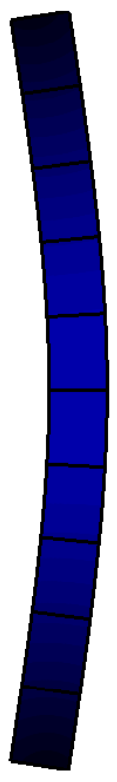

(e)

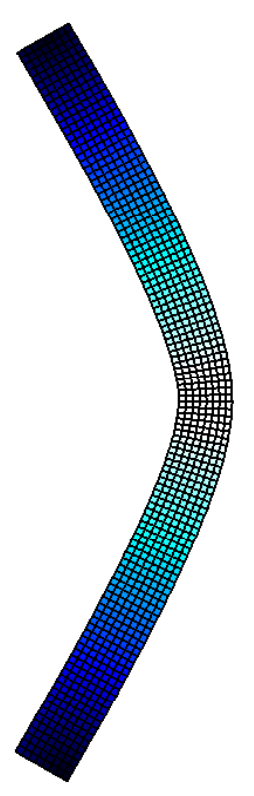

(b)

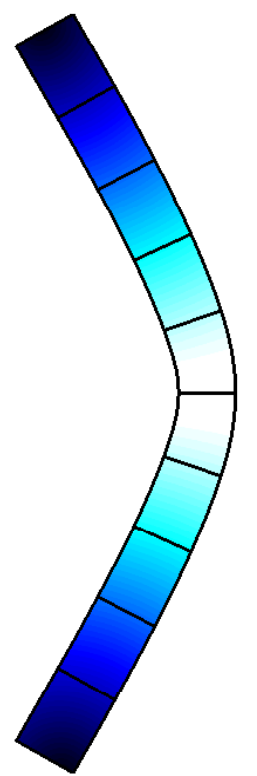

(f)
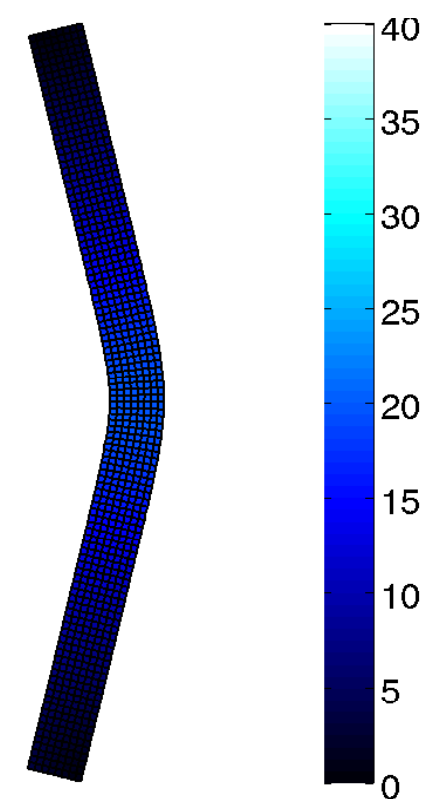

(c)

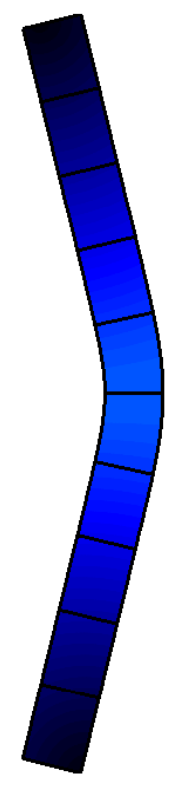

(g) (d)

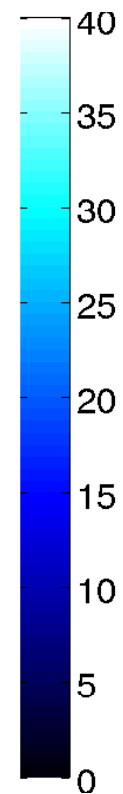

(h)

Fig. 12 Deformed meshes in pixel for three deformed images for a bidimensional analysis with 16-pixel element (top) and an analysis with beam elements using $C^{p-1}$ NURBS function with $p=5$ (bottom). 


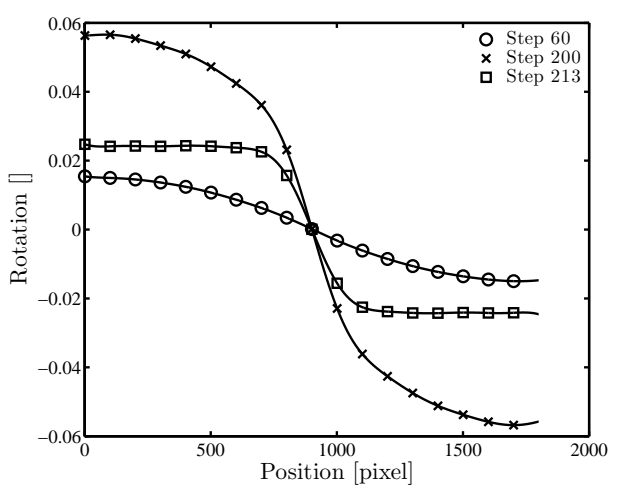

(a)

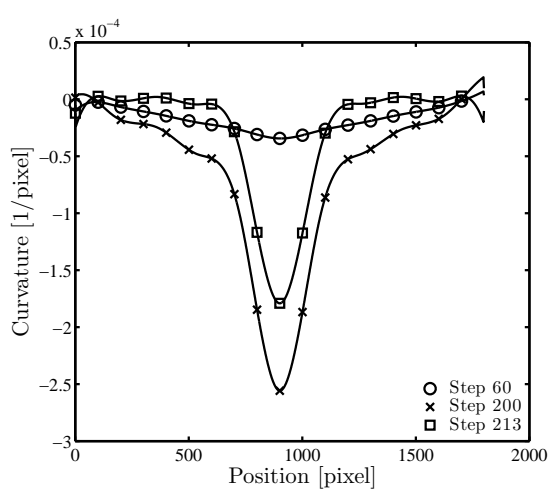

(b)

Fig. 13 Evolution of the rotation and curvature along the beam axis for 10 Euler-Bernoulli beam elements using NURBS functions with $p=5$. 


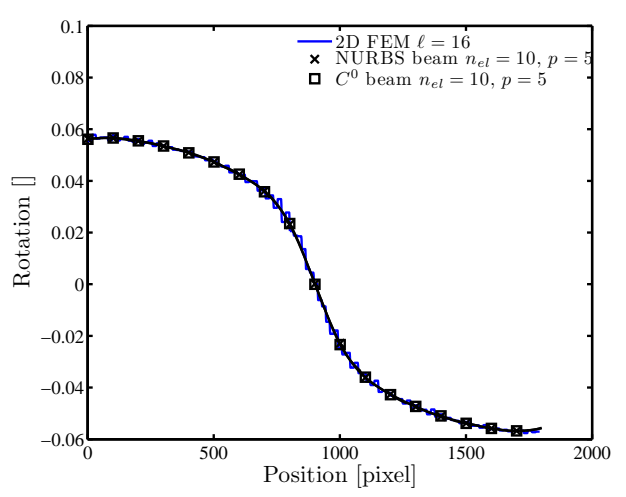

(a)

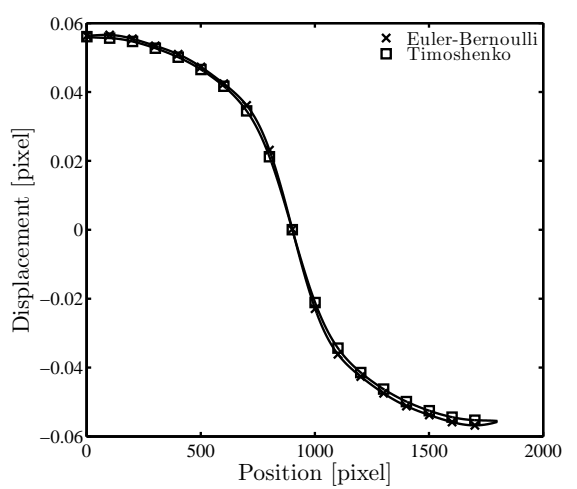

(c)

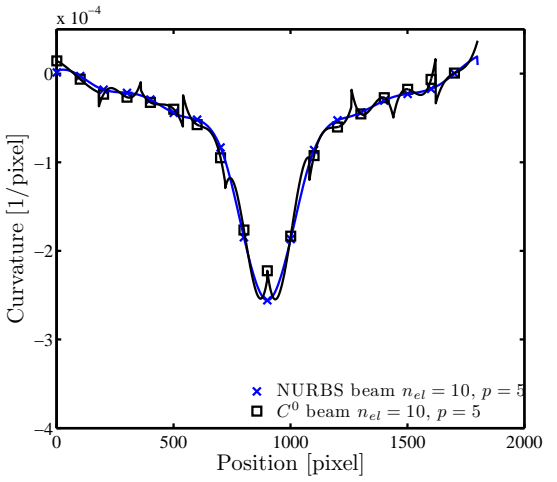

(b)

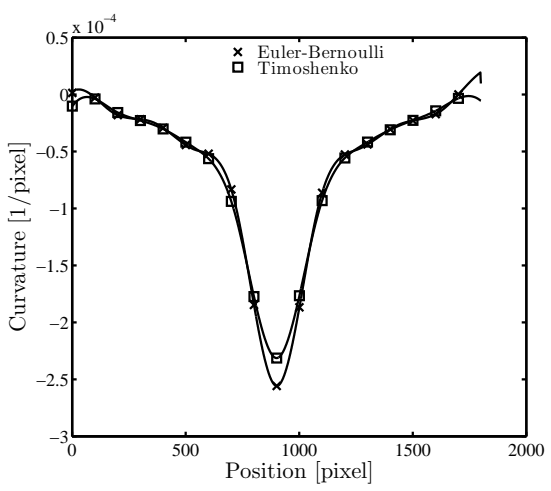

(d)

Fig. 14 Comparison of rotation (left) and curvature (right) for different discretizations (top) and for different beam models (bottom). 


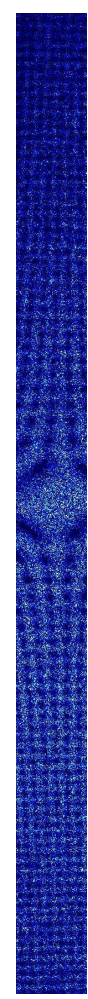

(a)

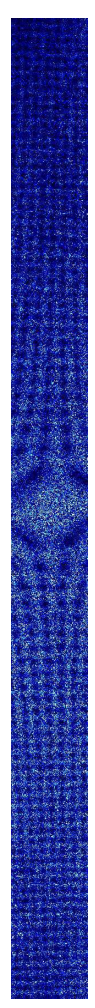

(b)

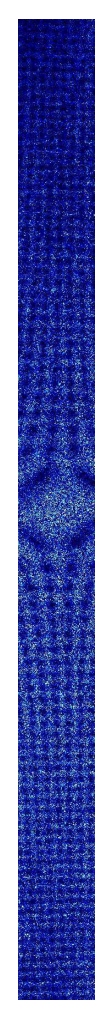

(c)

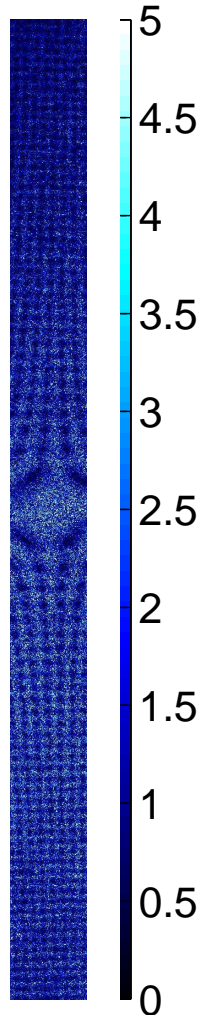

(d)

Fig. 15 Comparison of the error maps normalized by the dynamic of the initial image in percent for: (a) 2D FE with 16-pixel elements, (b) 10 Euler-Bernoulli beam elements using NURBS functions with $p=5$, (c) 10 Euler-Bernoulli beam elements using FE $C^{0}$ functions with $p=5$, (d) 10 Timoshenko beam elements using NURBS functions with $p=5$ 


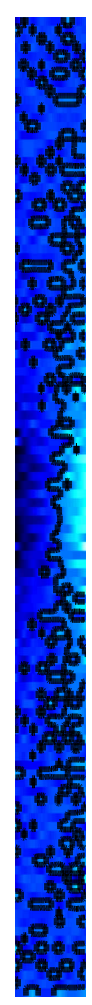

(a)

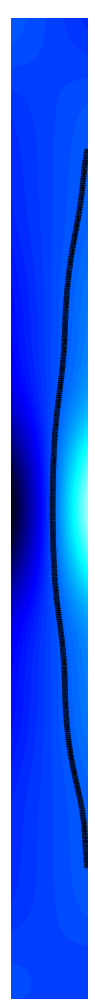

(b)

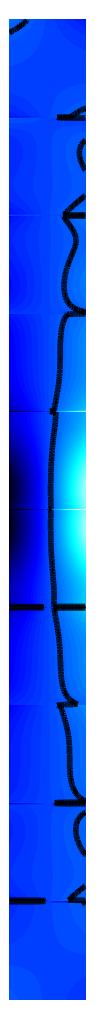

(c)

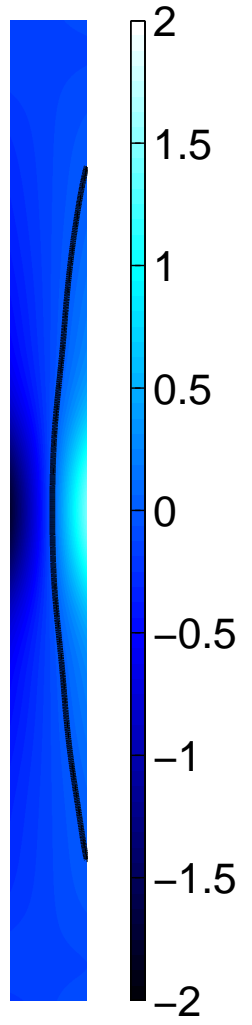

(d)

Fig. 16 Comparison of the axial strain $\varepsilon_{y y}$ in percent for: (a) 2D FE with 16-pixel elements, (b) 10 Euler-Bernoulli beam elements using NURBS functions with $p=5$, (c) 10 EulerBernoulli beam elements using FE $C^{0}$ functions with $p=5$, (d) 10 Timoshenko beam elements using NURBS functions with $p=5$. The black line is the iso- 0 of $\varepsilon_{y y}$. 